\title{
219. On Ergodic and Abelian Automorphism Groups of von Neumann Algebras
}

\author{
By Hisashi CHODA \\ Department of Mathematics, Osaka Kyoiku University \\ (Comm. by Kinjirô KUnUGI, M. J. A., Oct. 12, 1971)
}

Recently, in [5] Tam proved that any ergodic and abelian automorphism group of an abelian von Neumann algebra is freely acting.

In this paper, we shall give a generalization of Tam's theorem, using the notion of the generalized free action due to Kallman [3]. And we shall generalize Kallman's theorem that all the powers of an ergodic automorphism of a $\mathrm{II}_{1}$-factor are outer [3].

1. Let $\mathcal{A}$ be a von Neumann algebra acting on a Hilbert space $\mathfrak{F}$. In this paper we shall write briefly a ${ }^{*}$-automorphism of $\mathcal{A}$ as an automorphism of $\mathcal{A}$.

Definition A. Let $G$ be a group of automorphisms of a von Neumann algebra $\mathcal{A}$. Then $G$ is called to be ergodic on $\mathcal{A}$ if the only $A$ in $\mathcal{A}$ which satisfies

(*) $\quad g(A)=A \quad($ for all $g \in G)$

is scalar. An automorphism $g$ on $\mathcal{A}$ is called to be ergodic on $\mathcal{A}$ if the only $A$ in $\mathcal{A}$ which satisfies the condition (*) is scalar.

Kallman [3] has generalized the von Neumann free action for an abelian von Neumann algebra as follows:

Definition B (Kallman). An automorphism $g$ on a von Neumann algebra $\mathcal{A}$ is called to be freely acting on $\mathcal{A}$ if the only $A$ in $\mathcal{A}$ which satisfies

(**) $\quad A B=g(B) A \quad$ for all $B \in \mathcal{A}$

is $A=0$.

The condition $\left(^{* *}\right)$ in the Definition B is used by Nakamura and Takeda and plays an important role in the theory of the crossed product [4].

Under the Definition B, Kallman showed that any automorphism of a von Neumann algebra is decomposed into freely acting part and inner part. Using this theorem, we have the following:

Lemma 1. Let $\mathcal{A}$ be a von Neumann algebra, $G$ an ergodic group of automorphisms of $\mathcal{A}$ and $\alpha$ an automorphism of $\mathcal{A}$ such that

$$
\alpha g=g \alpha \quad \text { for every } g \in G \text {. }
$$

Then the automorphism $\alpha$ is freely acting or inner.

Proof. By the Kallman theorem, there exist a central projection $P$ and a unitary operator $U$ in $\mathcal{A}$ such that 
and that $\alpha$ is freely acting on $\mathcal{A}_{I-P}$.

$$
\alpha(A P)=U^{*} A P U \quad \text { for any } A \text { in } \mathcal{A},
$$

For any element $g$ of $G$,

and

$$
\alpha(g(P))=g(\alpha(P))=g(P)
$$

$$
\begin{aligned}
\alpha(g(P) B) & =g\left(\alpha\left(P g^{-1}(B)\right)\right) \\
& =g\left(U^{*} g^{-1}(B) P U\right) \\
& =g(U)^{*} B g(P) g(U) \quad \text { for any } B \text { in } \mathcal{A} .
\end{aligned}
$$

Then, by the definition of $P$ [3, Proof of Theorem 1], we have

$$
g(P) \leqq P \quad \text { for any } g \in G .
$$

On the other hand, since $G$ is ergodic,

$$
\sup \{g(P) ; g \in G\}=0 \quad \text { or } \quad I .
$$

Therefore $P=0$ or $I$, that is, $\alpha$ is inner or freely acting.

By Lemma 1, we have the following generalized Tom's theorem:

Theorem 2. Let $\mathcal{A}$ be a von Neumann algebra and $G$ an ergodic and abelian group of outer automorphisms of $\mathcal{A}$. Then $G$ is freely acting on $\mathcal{A}$.

Proof. For any element $g \in G(g \neq e$, unit of $G)$, we have

$$
g h=h g \quad \text { for any } h \in G \text {. }
$$

Then, by Lemma $1, g$ is freely acting or inner.

Therefore, since $G$ is a group of outer automorphisms of $\mathcal{A}, g$ is freely acting on $\mathcal{A}$. That is, $G$ is freely acting.

2. The following lemma may be known in the specialist.

Lemma 3. Let $A$ be a continuous von Neumann algebra acting on $\mathfrak{S}_{\mathcal{E}}$ and $\mathcal{B}$ a maximal abelian subalgebra of $\mathcal{A}$. Then for any nonzero projection $P$ in $\mathcal{B}$ there exist two orthogonal nonzero projections $Q$ and $R$ in $\mathscr{B}$ such that

$$
P=Q+R .
$$

Proof. It is sufficient to show that $\mathscr{B}$ does not have any minimal projection in $\mathscr{B}$. If there is a minimal projection $P$ in $\mathscr{B}$, then by the minimality of $P$, the reduced von Neumann algebra $\mathscr{B}_{P}$ is the algebra $\mathcal{C}_{P(\mathfrak{W})}$ of scalar multiples of the identity on $P\left(\mathfrak{S C}_{\mathfrak{S})}\right.$. On the other hand, since $\mathscr{B}$ is a maximal abelian subalgebra of $\mathcal{A}, \mathscr{B}_{P}$ is a maximal abelian subalgebra of $\mathcal{A}_{P}\left[1, \mathrm{p} .13\right.$ and p. 18]. Then $\mathcal{B}_{P}$ equals to $\mathcal{A}_{P}$ by the following equality ;

$$
\mathscr{A}_{P}=\mathcal{A}_{P} \cap \mathcal{C}_{P}{ }^{\prime}=\mathscr{A}_{P} \cap \mathscr{B}_{P}{ }^{\prime}=\mathscr{B}_{P} .
$$

This contradicts that $\mathcal{A}_{P}$ is continuous $[1$, p. 125].

It is known that all the powers of an ergodic measure preserving automorphism on a non-atomic probablility measure space are freely acting [2]. As an analogous statement for $\mathrm{II}_{1}$-factor, Kallman proved in [3] that all the powers of an ergodic automorphism of $\mathrm{II}_{1}$-factor are outer. We have a generalization of this theorem as follows : 
Theorem 4. Let $\mathcal{A}$ be a continuous von Neumann algebra acting on a Hilbert space $\mathfrak{S}$ and $g$ an ergodic automorphism of $\mathcal{A}$. Then $g^{n}(n= \pm 1, \pm 2, \ldots)$ is freely acting on $\mathcal{A}$.

Proof. If $g^{n}$ does not freely acting for some $n(= \pm 1, \pm 2, \cdots)$, then by Lemma $1 g^{n}$ is inner. We may assume that $n>0$. Then there exists a unitary operator $U$ in $\mathcal{A}$ such that

$$
g^{n}(A)=U^{*} A U \quad \text { for all } A \text { in } \mathcal{A} \text {. }
$$

Let $\mathscr{B}$ be a maximal abelian subalgebra of $\mathcal{A}$ containing $U$. Then we have

$$
g^{n}(B)=B \quad \text { for all } B \text { in } \mathscr{B} \text {. }
$$

Let, for any nonzero projection $Q$ in $\mathscr{B}$,

$$
R=Q+g(Q)+\cdots+g^{n-1}(Q) .
$$

Then $g(R)=R$, so $R$ is some scalar multiple of the identity, say $R=\lambda I$. Since $Q$ is a nonzero projection, we have $\lambda \geqq 1$.

Take a unit vector $x$ in $\mathcal{H}$. For a natural number $k$ with $k>n^{2}$, there exist $k$ mutually orthogonal projections $Q_{i}$ in $\mathscr{B}(i=1,2, \cdots, k)$ with $\sum_{i=1}^{k} Q_{i}=I$, by Lemma 3. By the equality;

$$
1=\|I x\|^{2}=\sum_{i=1}^{k}\left\|Q_{i} x\right\|^{2},
$$

there exists $i$ such as

$$
\left\|Q_{i} x\right\|<1 / n \text {. }
$$

If $\left\|g\left(Q_{i}\right) x\right\| \geqq 1 / n$, we choose again $k$ mutually orthogonal nonzero projections $R_{j}(j=1,2, \cdots, k)$ in $\mathscr{B}$ with $Q_{i}=\sum_{j=1}^{k} R_{j}$, by Lemma 3 . As such as $Q_{i}$,

$$
1 \geqq\left\|g\left(Q_{i}\right) x\right\|^{2}=\sum_{j=1}^{k}\left\|g\left(R_{j}\right)\right\|^{2},
$$

then there exists a nonzero projection $R_{j}$ in $\mathscr{B}$ with $\left\|g\left(R_{j}\right) x\right\|<1 / n$. Then we have a nonzero projection $Q$ in $\mathscr{B}$ such that

and

$$
\|Q x\|<1 / n
$$

$$
\|g(Q) x\|<1 / n \text {. }
$$

Going on this method, we have a nonzero projection $Q$ in $\mathscr{B}$ such as for any $k(1 \leqq k \leqq n)$,

$$
\left\|g^{k}(Q) x\right\|<1 / n
$$

Since, for this nonzero projection $Q$ in $\mathscr{B}$,

$$
\lambda x=R x=Q x+g(Q) x+\cdots+g^{n-1}(Q) x,
$$

$|\lambda|<1$ which contradicts $\lambda \geqq 1$.

By the proof of Theorem 4, we can see that Theorem 4 is valid for a nonatomic abelian von Neumann algebra.

The author wishes to express his hearty thanks to Dr. P. K. Tam for the opportunity to see a prepublication copy of [5]. 


\section{References}

[1] J. Dixmier: Les algèbres d'opérateurs dans l'espace Hilbertien. GauthierVillars, Paris (1957).

[ 2 ] H. A. Dye: On groups of measure preserving transformations. I. Amer. J. Math., 81, 119-159 (1959).

[3] R. R. Kallman: A Decomposition Theorem of Automorphisms of von Neumann Algebras. Proceedings of a Symposium on Functional Analysis (edited by C. O. Wilde), Academic Press, New York, 33-35 (1970).

[4] M. Nakamura and Z. Takeda: On some elementary properties of the crossed products of von Neumann algebras. Proc. Japan Acad., 34, 489494 (1958).

[5] P. K. Tam: On an ergodic abelian $M$-group. Proc. Japan Acad., 47, $456-457$ (1971). 